\title{
Depression and Diabetes: Common Link and Challenges of Developing Epidemic!!
}

\author{
Sheikh Shoib ${ }^{1^{*}}$, Raheel Mushtaq ${ }^{1}$, Arif Tasleem $^{2}$ and Bhat M Hayat ${ }^{1}$ \\ ${ }^{1}$ Foundation Department of Psychiatry, Government Medical College Srinagar, Srinagar, India \\ ${ }^{2}$ Department of Dermatology, STD and Leprosy, Government Medical College Srinagar, Srinagar, India
}

"Corresponding author: Sheikh Shoib, Resident, Trauma Clinic, Post Graduate Department of Psychiatry, Government Medical College, Sringar, India. Tel: 9797197388; E-mail: sheikhshoib22@yahoo.com

Received Date: June 03, 2014, Accepted Date: December 28, 2014, Published Date: January 04, 2015

Copyright: (c) 2015 Sheikh Shoib, et al. This is an open-access article distributed under the terms of the Creative Commons Attribution License, which permits unrestricted use, distribution, and reproduction in any medium, provided the original author and source are credited.

\begin{abstract}
Diabetes mellitus (DM) is a chronic debilitating disease, which can cause various psychological, emotional, social and psychosexual problems. Depression can be a risk factor as well as complication of diabetes because of its link with compliance issues, glycaemic control and increased risk of complications. Early recognition and treatment of depression can go a long way in improving the quality of diabetic patients and it can also improve medical outcome. The aim of the article is to get overview of the association of the two common disorders and knowledge of their association would add to the framework of the global campaign to reduce burden world-wide.
\end{abstract}

Keywords Major depressive disorder; Diabetes mellitus; Psychiatric morbidity

\section{Introduction}

Diabetes mellitus is a chronic debilitating illness, with prevalence estimates that are approaching epidemic proportion worldwide. From 1985 to 2000, the number of people living with diabetes globally has risen from 30 million to 171 million. 1An estimated 285 million people corresponding 6-8\% adult population will live with diabetes in 2010. The number is expected to increases to 438 million by $2030.1 \mathrm{With}$ an estimated 50.8 million people living with diabetes, India has the world's largest population, followed by china with 43.2 million with diabetes population. The recent increase of diabetes in India and projections for the future constitute a major public health problem $[1,2]$.

Psychological disturbances are commonly observed during the course of endocrine disorders. In recent years there has been an increased interest in the psychological wellbeing of patients suffering with endocrinological disorder. The relationship between endocrinological disorders particularly thyroid and diabetes continues to generate considerable interest in research [3,4]. Psychological distress is frequently seen in diabetes mellitus and usually occurs after the diagnoses. The quality of life of patient is adversely affected, due to the course of the illness, restriction of diet and activity, management schedules and its complications [2]. "Diabetes burnout" and "Diabetes overwhelm us" are the two words used often to describe the distress experienced by diabetic patients [2]. Diabetes burnout is the term given to state of disappointment that occurs after submission to diabetes. Self-destructive behaviors like over eating can occur during diabetic burnout .People who experience this burnout report seeking freedom from this frustrating condition and can accompany various psychological changes like anxiety and depression. The end results is aggravating the diabetes and causing complications [2].

Comorbid depression is common in chronic medical conditions including diabetes. Depression and diabetes are highly prevalent conditions and have significant impact on health outcomes [2] further, the prognoses of both depression and diabetes with respect to disease severity, complications, treatment resistance, and cost is worse for either disease than when they occur together. Given that comorbidity of disorders adds to treatment complexity and is frequently associated with chronicity, it is not surprising that diabetes mellitus negatively affects the course of psychiatric disorder [5]. The aim of our review on depression and diabetes was to emphasize the association of two in this part of the world, because of modern day epidemics of diabetes and obesity that is emerging in developing countries.

On other hand, major depressive disorder (MDD) is one of the most common psychiatric disorders with high prevalence worldwide [6] Approximately 340 million people worldwide suffer from depression at any given time. 10 to $25 \%$ of women and 5 to $12 \%$ of men have lifetime risk of developing major depressive disorder in community samples as per American Psychiatric Association, 2000 [7]. Prevalence rates appear to be unrelated to marital status, education, income, or ethnicity. According to world health organization, depression is responsible for greatest burden related to non-fatal health outcomes. Morbidity, mortality, disability, workplace absenteeism, lost work productivity, and increased use of health resources are the consequences of depression [8]. Major depressive disorder (MDD) is a multi-factorial disease, with both genetic and environmental factors likely to have a role in its etiology.

Diabetic patients are prone to develop varied psychosocial and emotional problems, due to which the quality of life in them is affected. Serious short term complications such as hypoglycemia, and disabling long term complications like cardiovascular disease, neuropathy, nephropathy and retinopathy occurs in patients with Diabetes [4,9]. Further, depression is common among diabetic patients, with co-morbid health problems, as compared to patients with diabetes alone. Depression usually leads to an unhealthy life style among patients with diabetes and that may be one of the major factors contributing to decreasing life span. The management of depression affects course and outcome of diabetes lead to improvement in glycaemic control [9]. 
Methodology: A review of literature regarding depression and diabetes has been done to assess the prevalence, pathogenesis, risk factors associated with diabetes. We reviewed the published literature on Diabetes and depression from 1993 to 2013. Our search strategy for this review involved two stages. Published studies were identified by using following keywords diabetes and depression in search of in various search engines like Medline and goggle scholar. References in the resulting articles were reviewed and relevant studies were included in this review.

\section{Prevalence of depression in Diabetes Mellitus}

Depression is a common medical disorder, which remains underrecognized and under-treated. The prevalence of depression in DM varies and depends on various factors [10]. Socioeconomic status, family status, obesity, smoking habits, psychical activity and sedentary life are the some of the important contributory factors. The occurrence of major depression in DM is mostly estimated to be around $12 \%$ (ranging from 8-18\%). 15-35 \% of individuals with DM report milder types of depression [10]. It is estimated that individual with DM are 1.4-3 times likely to suffer from comorbid depression. Prevalent of depression in DM particularly with respect to patients with other chronic diseases is debatable issue. Presence of psychiatric comorbidity in diabetes varies and depends on various measures .Those using self-report measures report higher prevalence than using semi-structured interviews. Selection biases are the main problem in small studies [11].

Various meta-analyses have been done and have reviewed the literature regarding this area. The meta-analyses by Gavard et al. analyzed 18 studies ( 7 controlled) and found mean prevalence of diabetes of $14 \%$ by diagnostics interview and $32 \%$ using self report symptoms scale in controlled studies and $15.4 \%$ and $19.6 \%$ in controlled [12]. Further, Anderson et al. reviewed 42 studies (20 controlled) and found elevated depressive symptoms: $31 \%$ controlled studies: $20,(5 \%$ vs $11.4 \%)$; OR=2.0 uncontrolled studies: $29.7 \%$ T1DM: $\mathrm{OR}=2.9$ (1.6-5.5), T2DM: $\mathrm{OR}=2.9$ (2.3-3.7) Overall: $\mathrm{OR}=2.0(1.8-2.2)$ independent of sex, DM type, sample source, depression assessment method.11 Another meta-analyses done by Barnard et al, (2006) analyzed T1DM 4 controlled \& 10 uncontrolled studies and found prevalence of depression as $12 \%$ vs $3.2 \%$ (controlled studies) $13.4 \%$ (uncontrolled studies). 13 One of the large cross-sectional communitybased study by done Kruse et al (2003) using Composite International Diagnostic Interview (CIDI) and found comparable levels of depression between patients with $\mathrm{DM}$ and non-diabetic individuals. [26.6\% vs 26.0\%; Odds Ratio $(\mathrm{OR})=1.11$; Confidence Interval $(\mathrm{CI})$ : 0.73-1.69] [14].

It is estimated that, depressions affects 1 in 8 of diabetic patients. However, 1 in 5 of the remaining patients shows symptoms of depression [15] As per recent research, depressive symptoms seem to be slightly more prevalent in Type 2 DM compared to Type $1 \mathrm{DM}$ though this difference is not regarded to be statistically significant. Further studies, comparing the prevalence of depression in samples including patients with diabetes of either type, also adjusting for potential confounders, as already discussed $[15,16]$.

\section{Double trouble (Diabetes and depression)}

The relationship between DM and depression seems to be bidirectional. Studies have reported that relative risk of developing T2DM in depressed patients (Depression $\rightarrow$ Diabetes) is as high as 1.621 and relative risk for developing depression in patients with DM (Diabetes $\rightarrow$ Depression) is 1.22 .17 Patients with diabetes and coexisting depression have higher odds of disability compared to those alone $[17,18]$ Figure 1 shows odds of disability in three groups of patients.
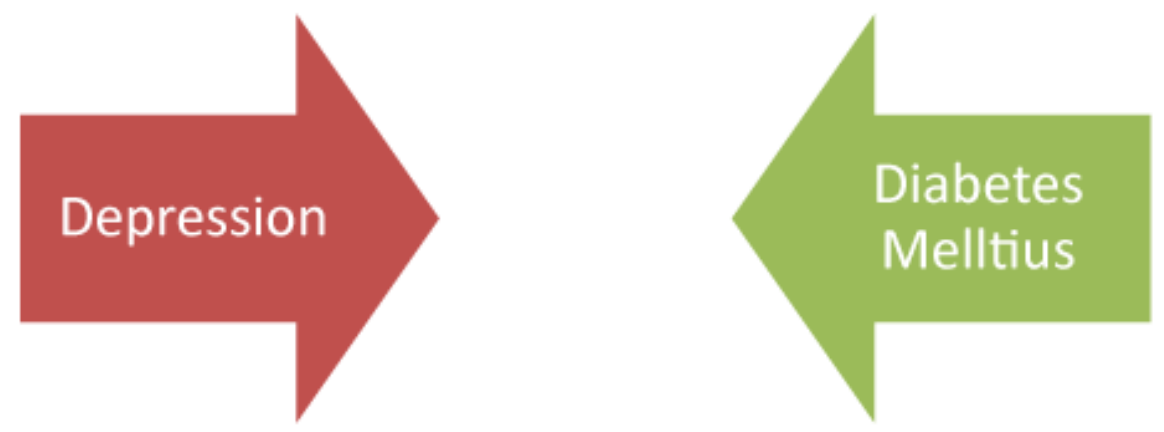

Figure 1: Odds of disability in three groups of patients.

\section{Risk factors for the development of depression in Diabetes Mellitius}

There are various risk factors associated with depression in diabetes. They include female sex, younger age, not having a spouse, poor social support, lower education and low socioeconomic status. History of poor glycemic control, diabetic complications, medical comorbidity, physical impairment and previous history of depression are the other risk factors for development of depression [19] A range of potential risk factors for depression in diabetes have been postulated . However, much of evidence of evidence cited above is from crosses sectional studies and the temporal relationship between diabetes and depression is yet to be fully determined $[19,20]$.

\section{Pathogenesis of diabetes-depression link}

The exact cause responsible for high prevalence of depression in depression and its effects on outcome is not well understood. However biological factors, psychological factors and their interaction are proposed to be causative agents for explaining this mechanism. Recent 
work on pathogenesis of depression-depression link has proven multiple interconnected mechanisms [21,22]. These include metabolic programming at genetic level and early childhood and utero nutrition, changing life styles both individually and collectively induce hyperactivity of HPA axis (hypothalamic-pituitary-adrenal axis). The end results in sympathetic nervous system activation and long standing hypercortisolaemia is suggested to be for its distinct process. It includes activation of cell mediated immune response, accumulation of visceral fat and insulin resistance, which ultimately progresses to DM [23].

Depression is likely to play role in several ways of pathogenesis. First as a coincidental consequence of same environmental factors, that influence glucose metabolism. Second as an independent factor that also affects nutrition and life style behavior. Third as an phenotype of stress related disorders which have unifying over activation of HPA and inflammatory response to stress and as a marker of severity of underling pathogenesis of diabetes. Over all, the evidence is pointing towards depression -diabetes link, having to some extent a common origin in which some individuals are more vulnerable and programmed such that there is over activation of those stress regulation pathway which contribute to metabolic dysregulation [24].

\section{Depression and insulin resistance}

The continuum of diabetes stretches from developing insulin resistance to impaired glucose intolerance and frank diabetes occurs as insulin secretion fails. Insulin resistance is regarded as the fundamental step connecting depression to diabetes and varied pathways exists in these mechanisms. The activation of hypothalamicpituitary adrenal (HPA) axis occurs in $40-60 \%$ of patients with major depressive disorder. Further causing increasing levels of cortisol and causing disturbances of glucoregulatory mechanisms. The end result is hyperinsulinemia and insulin resistance, eventually leading to frank diabetes. Changes in lifestyle factors associated with depression also increase the risk of developing insulin resistance. Obesity is a common pathway and is a significant risk factor for increasing both insulin resistance and diabetes [24,25].

There are meta-analysis of nine prospective epidemiological studies, which showed that depression is associated with an increased risk of type 2 diabetes. The first observational study was done by British Women's and Health study of 4286 women aged 60-79 years randomly selected from primary care. The association was between two disorders was almost $U$ shaped, with prevalence of depression decreasing linearly with increasing insulin resistance among women without diabetes and increasing among women with diabetes [26] Lawlor et al. reported that depression was inversely related to insulin resistance among 4,268 British woman aged 60-79 years. ( Odds Ratio(OR)=-0.84; Confidence Interval (CI):0.74-0.97, $\mathrm{p}=0.006$ ) [26]. Timonen et al. in a cross-sectional study comprising of 491 male and female patients. The study enrolled individuals aged 61-63 years and reported that insulin resistance and severity of depressive symptoms were positively correlated, particularly in people with IGT.( $r=0.13$, $\mathrm{p}=0.0004$ ) [27]. However, these studies are in contrast with Lawlor et al. who concluded that reduced depressive symptoms are not associated with insulin resistance among 2,512 Welsh men aged 45-59 years. $(\mathrm{OR})=-0.97$; (CI): 0.77-1.23, p not significant ) [28]. These large difference in results can be explained by difference in the type of study (longitudinal vs cross-sectional) and patient samples (men vs women).However it is important clear that association between depression with insulin resistance is different between men and women and across glucose tolerance status [29].

\section{Inflammation, insulin resistance and diabetes}

Recent research has shown that there is increased evidence between chronic inflammation and insulin resistance. There is also a growing body of consensus over the last decade on characteristics of inflammation in obesity and the mechanisms by which this inflammation contributes to insulin resistance. It is now suggested that inflammation in diabetes and insulin resistance occurs through inhibition of insulin receptor signalling pathways [30]. Among the proinflammatory cytokine TNF-a mediate insulin resistance as a result of obesity in many rodent obesity models. Chemokine monocyte chemo tactic protein-1 (MCP-1) is another newcytokine proposed for impair adipocyte insulin sensitivity [31] shown in Figure 2.

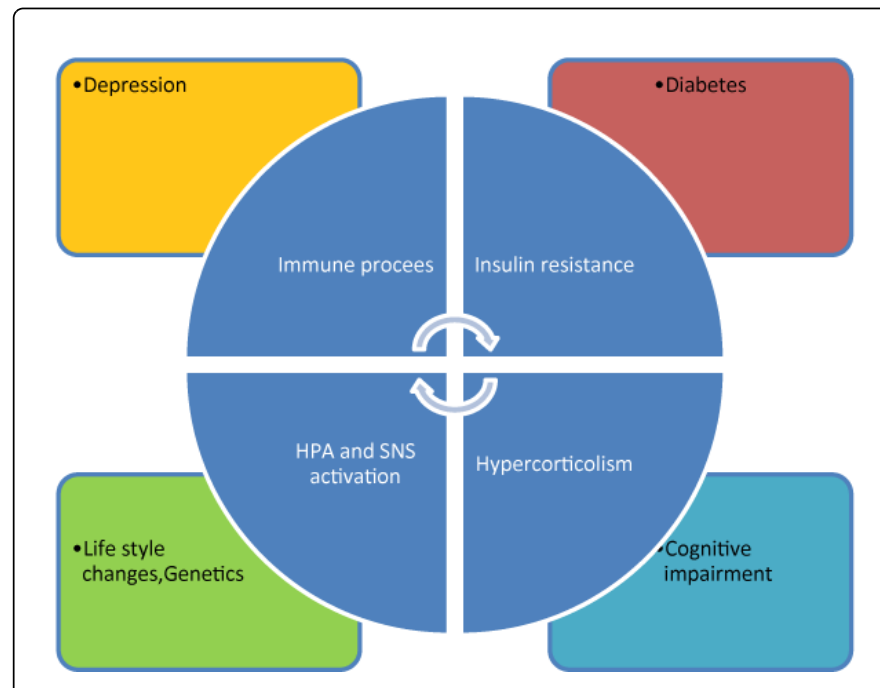

Figure 2: Inflammation, insulin resistance and diabetes

\section{Impact of Depression on Diabetes}

The co morbid diabetes and depression is associated with significant morbidity as well as mortality, therefore focusing and treating depression is very important and needs special attention $[32,33]$. There is a close relationship between distressing life events in diabetic and period of diabetic control, which may even lead to development of ketotic coma. Patients treated for diabetes have higher chances of developing depressive symptoms, during follow up period. Further, when depressive symptom occurs, they are associated with poorer adherence to diabetic diet, poorer compliance to drugs, complications and impairment in function [33,34]. This fact is supported by at least 3 randomized control trials. The three trials have shown that treatment either with antidepressants or cognitive behavior therapy results in improvement in glycemic level as well as mood symptoms. Whatever the underlying exact causes are, depression may have an important bearing on course of diabetes, leading to worsening of diabetes control and increased complications. Effective treatment facilitates compliance to diet and exercise and results in improving glycaemic control [35-37]. 


\section{Management of depression and diabetes}

The proper management of depression in individuals with diabetes depends on integrated treatment as well as overall care of the patients. There is a general consensus of close relationship between depression, diabetes and further each of these two conditions can effect patients overall health and course of the disease [38]. This is also supported by bidirectional relationship of depression and diabetes as each condition may increase the both risk and severity of other condition [39]. There is also the cost of care related to diabetes and depression is also greatly increased through the interaction of both two disorders. The effect of depression on management of diabetes is vast and complex. Depression affects quality of life and thus affects the life style activities, leading to changes in self-monitoring and medication usage. The cognitive dysfunction in depression patients also adds to the complexity of care and cost of care [40,41]. Depressions in diabetic patients have higher levels and increased severity of symptoms leading to more prolonged. Thus successful management of both disorders depends upon on integrated cooperation between different branches of medical sciences for optimal results for these disorders [41].

Varied factors influence treatment of depression in diabetes. Effective non pharmacological interventions such as regular exercise for depression, Cognitive behavioral therapy and psycho education should be offered [42]. Diabetics' patients suffering from mild depression, the initial treatment to offer is support to patient and their families followed by information regarding diseses. Problem solving therapy, cognitive therapy or interpersonal psychotherapy can be used in patients with moderate depression $[42,43]$.

\section{Antidepressants and Diabetes mellitius}

Antidepressants increase the risk of DM up to two fold. This risk is associated with both with tricyclic antidepressants and selective serotonin inhibitors (SSRI) and varies with duration of use of antidepressants. Antidepressant treatment for shorter periods or with lower daily doses was not associated with an increased risk $[44,45]$. Treatment of depression with selective serotonin reuptake inhibitors may improve glycemic control and may be beneficial for these patients. Among SSRI'S, Fluxetine has been shown to improve in HbAlc, decreases insulin requirements, loss of weight and increases insulin requirements. TCA causes increased weight gain, hyperglycemias and increased appetite [46]. Among TCA's nortrypline improves depression but worsens glycemic control in diabetes in study done by Lustman PJ et al. SSRI's does not disturb glycemic control and has seen has very less effect on weight gain and glycemic level .Among SNRI'S, dulextine has shown to no influence on glycemic control. Limited data are available on venlafaxine [47]. Although mirtapine is associated with weight gain, little data is available which shows its effect in diabetes. There is no data currently available with trazadone and robextine [48].

\section{Protective measures}

A multidisciplinary team approach, including an endocrinologist, diabetes training, and mental health practitioner, is ideal for the treatment of depression in DM. Depending on the severity of the comorbid psychopathology, a psychiatrist may also be needed for psychopharmacological evaluation and treatment [35]. Early on in treatment, intensive glycemic management of diabetes is an appropriate target for a person with diabetes and comorbid depression. The first goal should focus on medical stabilization.
Psychiatric interventions like education, counseling and treatment could be the missing link in the overall management of the patients suffering from diabetes mellitus $[35,36]$.

\section{Conclusion}

The presence of depression in diabetes has major consequences. Depression adds to treatment complexity, is frequently associated with chronicity and negatively affects the course of diabetes. Therefore, regular screening of depression should be integrated in standard diabetes care and can go a long way in improving the quality of life of these patients. Further community based studies are required to assess the magnitude of the comorbidity and improving the management of these patients.

\section{References}

1. Ahmad J, Shoib S, Ashraf M, Masoodi MA, Ahmad R, et al. (2012) Glucose Intolerance in Relation to Socioeconomic status and gender in population of Srinagar City. J Postgrad Med Inst 26: 352-5.

2. Dar MM, Shoib S, Ahangar WH, Bhat MY, Bashir H (2013) Psychiatric Aspects of Diabetes Mellitus-Hospital Based Study. IJHSR. 3: 70-79.

3. Dar MM, Shoib S, Bashir H (2013) Link between psychiatric and autoimmune thyroid disorder. Int J Health Sci Res 3: 30-37.

4. Shoib S, Mushtaq R, Dar MM (2013) Psychiatric Manifestations in thyroid disorders IJCCI 5: 84:98.

5. Gavard JA, Lustman PJ, Clouse RE (1993) Prevalence of depression in adults with diabetes: an epidemiological evaluation. Diabetes Care 16: 1167-1178.

6. Murray CJ, Lopez AD (1996) Evidence-based health policy-lessons from the Global Burden of Disease Study. Science 274: 740-743.

7. Angst J (1997) Epidemiology of depression. In Honig A, van Praag HM, eds. Depression: Neurobiological, Psychopathological and Therapeutic Advances. Chichester, UK: John Wiley.

8. Fombonne E (1999) True trends in affective disorders. In: Cohen P, Slomkoski C, Robins LN, eds. Historical and GeographicalInfluences on Psychopathology. New Jersey: Laurence Erlbaum 115-39.

9. Lin EH, Rutter CM, Katon W, Heckbert SR, Ciechanowski P, et al. (2010) Depression and advanced complications of diabetes: a prospective cohort study. Diabetes Care. 33: 264-269.

10. Gonzalez JS, Fischer L, Polonsky W (2011) Depression in diabetes: Have we been missing something important? Diabetes Care 34: 236-239.

11. Anderson RJ, Freedland KE, Clouse RE, Lustman PJ (2001) the prevalence of comorbid depression in adults with diabetes. A metaanalysis. Diabetes Care 24: 1069-1078.

12. Gavard JA, Lustman PJ, Clouse RE (1993) Prevalence of depression in adults with diabetes. An epidemiological evaluation. Diabetes Care 16: 1167-1178.

13. Barnard KD, Skinner TC, Peveler R (2006) the prevalence of co-morbid depression in adults with Type 1 diabetes: systematic literature review. Diabet Med 23: 445-448.

14. Kruse J, Schmitz N, Thefeld W (2003) On the association between diabetes and mental disorders in a community sample. Results from the German National Health Interview and Examination Survey. Diabetes Care 26: 1841-1846.

15. Anderson RJ, Freedland KE, Clouse RE,Lustman PJ (2001) The prevalence of comorbid depression in adults with diabetes: a metaanalysis. Diabetes Care 24:1069-1078.

16. Katon WJ, Von Korff M, Lin EH, Simon G, Ludman E, et al. (2004) The Pathways Study. a randomized trial of collaborative care in patients with diabetes and depression. Arch Gen Psychiat 61: 1042-1049.

17. Egede LE, Ellis C (2010) Diabete and depression: Global perspective. Diabetes research and clinical practice 87: 302-312. 
18. Knol MJ, Twisk JWR, Beekman ATF, Heine RJ, Snoek FJ, et al. (2006) Depression as a risk factor for the onset of type 2 diabetes mellitus. A meta-analysis. Diabetologia 49: 837-845.

19. Tellez-Zenteno JF, Cardiel MH (2002) Risk factors associated with depression in patients with type 2 diabetes mellitus. Arch Med Res 33 : 53-60.

20. Nouwen A, Winkley K, Twisk J, Lloyd CE, Peyrot M, et al. (2010) Type 2 diabetes mellitus as a risk factor for the onset of depression: a systematic review and meta-analysis Diabetologia 53: 2480-2486.

21. Eaton WW, Mengel M, Mengel L, Larson D, Campbell R, et al. (1992) Psychosocial and psychopathologic influences on management and control of insulin-dependent diabetes. Int J Psychiatry Med 22:105-117.

22. Brown ES, Varghese FP, Mc Ewen BS (2004) Association of depression with medical illness: does cortisol play a role? Biol Psychiatry 55: 1-9.

23. Weber-Hamann B, Hentschel F, Kniest A, Deuschle M, Colla M, et al. (2002) Hypercortisolemic depression is associated with increased intraabdominal fat. Psychosom Med 64: 274- 277.

24. Adriaanse MC, Dekker JM, Nijpels G, Heine RJ, Snoek FJ, et al. (2006) Associations between depressive symptoms and insulin resistance: the Hoorn Study. Diabetologia 49: 2874-2877.

25. Anagnostis P, Athyros V, Tziomalos K, Karagiannis A, Mikhailidis D (2009) The Pathogenetic Role of Cortisol in the Metabolic Syndrome: A Hypothesis The Journal of Clinical Endocrinology \& Metabolism 94: 2692-2701.

26. Lawlor DA, Smith GD, Ebrahim S (2003) Association of insulin resistance with depression: cross sectional findings from the British Women's Heart and Health Study. Br Med J 327: 1383-1384.

27. Timonen M, Laakso M, Jokelainen J, Rajala U, Meyer-Rochow VB, et al. (2005) Insulin resistance and depression: cross sectional study. Br Med J 330: 17-18.

28. Lawlor DA, Ben-Shlomo Y, Ebrahim S, Davey Smith G, Stansfeld SA, et al. (2005) Insulin resistance and depressive symptoms in middle aged men: findings from the Caerphilly prospective cohort study. BMJ 330: 705-706.

29. Pouwer F, Snoek FJ (2001) Association between symptoms of depression and glycaemic control may be unstable across gender. Diabet Med 18:595-598

30. Hotamisligil GS, Spiegelman BM (1994) Tumor necrosis factor alpha: a key component of the obesity-diabetes link. Diabetes. 43: 1271-1278.

31. Moller DE (2000) Potential role of TNF-alpha in the pathogenesis of insulin resistance and type 2 diabetes. Trends Endocrinol. Metab. 11: 212-217.

32. Kohen D, Burgess AP, Catalán J, Lant A (1998) the role of anxiety and depression in quality of life and symptom reporting in people with diabetes mellitus. Qual Life Res 7: 197-204.

33. Goldney RD, Phillips PJ, Fisher LJ, Wilson DH (2004) Diabetes, depression, And quality of life: a population study. Diabetes Care 27: 1066-70.
34. Ciehanowski P, Katon WJ, Russo JE (2000) Depression and diabetes; impact of depressive symptoms on adherence, function and cost. Archives of internal medicine : 1160: 3278-3285.

35. Lustman PJ, Anderson RJ, Freedland KE, De Groot M, Carney RM,et al. (2000) Depression and poor glycemic control ; a metanalytic review of literature. Diabetes care 23: 434-442.

36. Lustman PJ, Griffith LS, Freedland KE, Kissel SS, Clouse RE (1998) Cognitive behavioural therapy for depression in type 2 diabetes ; results of randomised control clinical trials . Annals of internal medicine 129: 613-621.

37. Lustman PJ, Griffith LS, Clouse RE (1997) Effects of nortriypline on depression and glycemic control in diabetes. Results of double blind placebo controlled clinical trials. Psychosomatic Medicine 59: 241-250.

38. Katon W (2003) Clinical and health services relationship between major depression, depressive symptoms and general medical illness. Biol Psychiatry 54: 216-226.

39. Golden Sh, Lazo M, Carnethon M (2008) Examining a bidirectional association between depressive symptoms and diabetes. JAMA: 299: 2751-2759.

40. Van der Feltz-Cornelis CM, Nuyen J, Stoop C, Chan J, Jacobson AM, et al. (2010) Effect of interventions for major depressive disorder and significant depressive symptoms in patients with diabetes mellitus: a systematic review and meta-analysis. Gen Hosp Psychiatry 32: 380-395.

41. Munshi M, Grande L, Hayes M (2006) Cognitive dysfunction is associated with poor diabetes in older adults. Diabete care: 29: 1797-1799.

42. Otto MW, Power M, Smits JAJ (2005) Adding Cognitive-Behavioral Therapy to Pharmacotherapy for Panic Disorder: Issues and Strategies.CNS Spectr 9: 32-39.

43. Popkin M, K Callies A, Mackenzie TB (1985) The Outcome of Antidepressant Use in the Medically Ill. Archives of General psychiatry 42: 1160-1163.

44. Kivim"aki M, Hamer M, Batty GD, Geddes JR, Tabak A G, et al. (2010) Antidepressant Medication Use, Weight Gain, and Risk of Type 2 Diabetes. Diabetes Care 33: 2611-2616.

45. Lustman PJ, Griffith LS, Clouse RE, Freedland KE, Eisen SAet al. (1997) Effects of nortriptyline on depression and glycemic control in diabetes: results of a double-blind, placebocontrolled trial. Psychosom Med 59: 241-250.

46. Maheux P, Ducros F, Bourque J, Garon J, Chiasson JL (1997) Fluoxetine improves insulin sensitivity in obese patients with non-insulindependent diabetes mellitus independently of weight loss. Int J Obes Relat Metab Disord 21: 97-102.

47. Raskin J, Smith TR, Wong K, Pritchett YL, D'Souza DN, et al. (2006) Duloxetine versus routine care in the long-term management of diabetic peripheral neuropathic pain. J Palliat Med 9: 29-40.

48. Himmerich H, Fulda S, Schaaf L Beitinger P, Schuld A, Pollma T (2006) Changes In Weight And Glucose Tolerance During Treatment With Mirtazapine Diabetes Care 29. 